\title{
RCMV increases intimal hyperplasia by inducing inflammation, MCP-1 expression and recruitment of adventitial cells to intima
}

\author{
Monika K Grudzinska ${ }^{1 *}$, Krzysztof Bojakowski ${ }^{2}$, Joanna Soin ${ }^{3}$, Frank Stassen ${ }^{4}$, Cecilia Söderberg-Nauclér ${ }^{* \dagger}$, \\ Piotr Religa ${ }^{1+}$
}

\begin{abstract}
Background: Cytomegalovirus (CMV) infection has been associated with accelerated transplant vasculopathy. In this study, we assessed the effects of acute rat CMV (RCMV) infection on vessel remodeling in transplant vasculopathy, focusing on allograft morphology, inflammation and contribution of adventitial cells to intimal hyperplasia.
\end{abstract}

Methods: Infrarenal aorta was locally infected with RCMV and transplanted from female F344 rats to male Lewis rats. Graft samples were collected 2 and 8 weeks after transplantation and analyzed for intimal hyperplasia, collagen degradation and inflammation. Transplantation of aorta followed by transplantation of RCMV infected and labeled isogenic adventitia were performed to study migration of adventitial cells towards the intima.

Results: Intimal hyperplasia was increased threefold in infected allografts. RCMV induced apoptosis in the media, expression of matrix metalloproteinase 2, and decreased collagen deposits. Macrophage infiltration was increased in the infected allografts and resulted in increased production of MCP-1. RCMV-infected macrophages were observed in the adventitia and intima. Cells derived from infected adventitia migrated towards the intima of the allograft.

Conclusions: RCMV enhances infiltration of macrophages to the allografts, and thereby increases MCP-1 production and inflammation, followed by recruitment of adventitial cells to the intima and accelerated intimal hyperplasia.

\section{Background}

Cytomegalovirus (CMV) is a well-known risk factor for late allograft dysfunction and is also associated with atherosclerosis and restenosis after angioplasty [1-6]. The major cause of late organ dysfunction after transplantation is accelerated transplant vasculopathy (TV), characterized by diffuse concentric intimal proliferation that results in vessel occlusion.

In the early phase of $\mathrm{TV}$, injured vessels contain predominantly macrophages and a few subendothelial lymphocytes ( $T, B$, and natural killer cells); late vascular lesions are associated with a thickened intima containing cells of

\footnotetext{
* Correspondence: Monika.Grudzinska@ki.se; Cecilia.Naucler@ki.se

+ Contributed equally

'Experimental Cardiovascular Research Unit, Department of Medicine, Solna, Karolinska Institutet, Stockholm, Sweden

Full list of author information is available at the end of the article
}

smooth muscle cell (SMC) phenotype interspersed with macrophages [7]. Intimal lesions are thought to be populated by dedifferentiated SMCs or vascular progenitor cells, either circulating or resident in the vessel wall, e.g. in the adventitia [8]. Furthermore, evidence has recently emerged suggesting that a vascular adventitia is activated in a variety of vascular diseases and plays an important role in the progression of vascular inflammation $[9,10]$. The number of progenitor cells contributing to vascular remodeling is thought to increase with the inflammatory response and their migration to sites of vascular injury is mediated by factors such as monocyte chemoattractant protein 1 (MCP-1) [11].

Clinical data and animal studies suggest that CMV contributes to the development of TV [12]. CMV infection of macrophages and endothelial cells affects cellular 
processes that may contribute directly to vascular disease. For example, CMV antigens activate the immune system [13] and hence may drive an inflammatory process in the arterial wall [14-17]. It is hypothesized that a local injury (e.g., allospecific injury or balloon angioplasty) can reactivate latent CMV in cells in the vessel wall or in cells recruited to the site of injury [2,18-20]. The virus then initiates an acute infection and inflammation, which may affect the migration and proliferation of vascular cells. In vitro, human CMV (HCMV) infection mediates vascular SMC migration that is dependent on the expression of the virally encoded chemokine receptor homologue US28 [21]. Deletion of the US28 rat homolgue $\mathrm{R} 33$ reduces the capacity of CMV to accelerate chronic rejection and TV in a rat model [22].

The mechanisms of HCMV-associated TV are difficult to determine because of its multifactorial etiology. Moreover, HCMV is ubiquitous and infects multiple cell types, including SMCs, endothelial cells, macrophages, and fibroblasts, and a primary infection is followed by lifelong latency, which makes it difficult to establish a specific temporal relationship between infection and TV [5]. Therefore, animal models are ideal for studying the association between CMV and TV.

The rat cytomegalovirus (RCMV) model has been useful to study mechanisms of CMV-related diseases, including the effect of the virus on TV [5,22-27]. In immunocompromised rats, RCMV causes a widespread infection of most tissues and various cell types, including endothelial cells, epithelial cells, macrophages, polymorphonuclear cells, and fibroblasts [28]. However, the effects of CMV infection on TV in rats and mice have so far only been studied after systemic infection [5,23-25,29-32].

To investigate the impact of a local CMV infection on cellular activation and vascular graft morphology in TV, we used a rat model in which the aorta or adventitia were locally infected with RCMV ex vivo after collection from the donor rat and before transplantation into the recipient rat. This model aims to mimic the effects of a severe systemic infection on the allograft and gives insight into which cells in the allograft become infected, and enable investigation of the cellular immune response against the virus and its impact on vascular remodelling.

\section{Methods}

\section{Ethics statement}

All animal procedures were approved by ethical committees of Stockholm North Ethical Committee and performed in accordance with institutional guidelines and conformed to the Guide for the Care and Use of Laboratory Animals at Karolinska Institute, Stockholm, Sweden.

\section{RCMV propagation}

RC 127 (Maastricht strain, $2.1 \times 10^{6} \mathrm{pfu} / \mathrm{ml}$; multiplicity of infection, $3.5 \times 10^{-2}$ ) was originally isolated from wild rats [33]. RCMV was propagated by infecting fibroblasts prepared from 17-day-old DA rat embryos. The supernatant was used for in vivo infection. Fibroblasts were cultured in flasks containing modified Eagle's minimum essential medium (Flow Laboratories) supplemented with $200 \mathrm{mmol} / \mathrm{l} \mathrm{L}$-glutamine (Northumbria Biologicals), antibiotic solution (10,000 IU/ml penicillin and 1000/ig/ $\mathrm{ml}$ streptomycin) (Gibco), and 10\% FCS (Sera-Lab) at $37^{\circ} \mathrm{C}$ in a $5 \% \mathrm{CO}_{2}$ incubator. At confluency, the cells were infected with RCMV according to standard viral culture techniques [34] and maintained in culture medium (as above) supplemented with 2\% FCS (Sera-Lab). After 5 to 7 days, when a cytopathic effect was observed in at least $95 \%$ of the cells, the cells were detached from the bottom of the flask by tapping and thereafter centrifuged at $1200 \mathrm{rpm}$ for $5 \mathrm{~min}$. The supernatant containing free RCMV was collected and immediately stored at $-70^{\circ} \mathrm{C}$. The virus was a gift from Prof. Cathrien A. Bruggeman's laboratory.

\section{Animal transplant models of locally infected with RCMV aorta and adventitia and study design}

Inbred male (150-170 g) Lewis rats (LEW.RT1 strain) and female Fisher rats (F344.RT1v1 strain) were used.

Rats used for aorta transplantation study $(\mathrm{n}=96)$ were divided into two groups: allograft (F344 to LEW) and isograft (LEW to LEW). The allograft group was divided into two experimental groups ( $\mathrm{n}=12$ rats each): (1) RCMV-infected allografts collected after 2 weeks (6 grafts); (2) RCMV-infected allografts collected after 8 weeks (6 grafts); and two control groups ( $n=12$ rats each), (3) uninfected allografts collected after 2 weeks (6 grafts), and (4) uninfected allografts collected after 8 weeks (6 grafts). Uninfected and infected isogenic grafts, isografts, ( $n=12$ rats in each group) were used as controls and were included to certify that there was no intimal hyperplasia in the isografts as was previously reported $[27,31,35]$. Transplantations of the infrarenal rat aorta: allograft (F344/LEW rats) and isograft (LEW/ LEW rats) were performed as described [36]. A part of the infrarenal aorta was taken from the donor, incubated for 20 min with $2.1 \times 10^{6}$ pfu RCMV (tissue culturederived virus diluted in $1 \mathrm{ml}$ of PBS), and transplanted into recipient rats. Allograft and isograft samples were collected 2 and 8 weeks post transplantation.

Thirty-six rats were used for adventitia transplantation. 12 rats (F344) served as donors of aorta, 12 rats of LEW strain served as donors of adventitia, and 12 rats of LEW strain served as recipients of both aorta and adventitia. Four weeks after aortic abdominal allograft transplantation (F344 to LEW), isogenic adventitial cells 
(LEW to LEW) labeled with cell tracker, which does not leak to adjacent cells (Molecular Probes, Carlsbad, CA), were transplanted to the allograft by cuffing labeled adventitia around the previously transplanted allograft. Allograft samples were collected 2 weeks post adventitia transplantation and studied using confocal microscopy for the migration of adventitial cells to the intima in vivo.

\section{Histologic and morphometric analyses of the allografts}

Transplanted aortas were collected, rinsed with $0.9 \%$ sodium chloride, fixed in $3 \%$ buffered formaldehyde for $4 \mathrm{~h}$, and stored at $4^{\circ} \mathrm{C}$ in PBS with $0.02 \%$ sodium azide. Thereafter, paraffin blocks were made and allografts were cut to obtain transverse sections at $5 \mu \mathrm{m}$. The sections were stained with hematoxylin and examined with a Leica light microscope. Intima and media crosssectional areas were measured with LeicaQWin software. The analysis was performed in a blinded approach by two observers who were unaware of coding.

\section{Immunohistochemistry of cross sections of the allografts}

The following primary antibodies were used for immunohistochemistry: mouse anti-human SM $\alpha$-actin, which is known to stain rat SM $\alpha$-actin [37] (Dako), mouse antirat CD45 (BD Pharmingen), mouse anti-rat CD68 (Serotec), mouse anti-rat CD3 (GeneTex), mouse anti-rat caspase 3 (Neomarkers), mouse monoclonal antibodies against MMP-2 and MMP-9 (Abcam), mouse anti-rat MAb8 against RCMV early/late antigen (R44-encoded protein, gift from C. Bruggeman), and mouse anti-MCP1 (Biolegend). Briefly, 5- $\mu$ m paraffin sections were deparaffinized, hydrated, and digested with pepsin. The sections were then subjected to antigen retrieval in citrate buffer followed by peroxidase (Innovex Sciences), avidin, and biotin (Dako) and Fc receptor blockage (Innovex Sciences). Sections were incubated with monoclonal antibodies, and the signal was visualized with a horseradish peroxidase detection system (BioGenex) using diaminobenzidine (Innovex Sciences) as the chromogen. For double immunohistochemistry, the signal was detected with Vector alkaline phosphatase using BCIP/NBT as substrate. After counterstaining with hematoxylin (Sigma-Aldrich), specimens were mounted in permanent mounting medium (Histolab).

\section{Masson's trichrome staining of cross sections of the allografts}

RCMV-infected and uninfected rat allografts collected at 2 and 8 weeks after transplantation were stained with Masson's trichrome stain to identify collagen deposits in the media as described [38]. Collagen deposits were assessed by computer-assisted histomorphometric image analysis in Adobe Photoshop. To estimate collagen content in the specimen, blue-stained areas were selected and quantified by an image-analysis system. The ranking procedure was performed in a blinded approach by two observers who were unaware of the coding.

\section{Statistics and data analysis}

The analysis was done on the cross sections of the allografts. The numbers of macrophages, lymphocytes, SMCs and apoptotic SMCs among all intimal and medial cells were determined by manual counting of cells positive for CD68, CD45, CD3, SM- $\alpha$ actin and cells double positive for caspase $3 / \mathrm{SM}-\alpha$-actin. RCMVinfected macrophages were quantified by calculating the percentage of cells double positive for late/early RCMV R44 protein and CD68 among all CD68-positive cells. MMP content was determined as the area immunostained, expressed as a percentage of the total area. Collagen deposits (blue-stained areas) and MCP-1 content (green-stained areas) were assessed by computerassisted histomorphometric image analysis in Adobe Photoshop. All ranking procedures were performed in a blinded approach by two observers who were unaware of coding. Results in multiple groups were analyzed with a two-tailed t-test, where the sample mean of the data obtained from infected allografts was compared to uninfected ones ( 2 weeks infected vs. 2 weeks uninfected, and 8 weeks infected vs. 8 weeks uninfected). P value $<0.05$ was considered as significant.

\section{Results}

RCMV infection of aortic allografts enhances intimal hyperplasia and media destruction, and decreases extracellular matrix deposition in the allograft

To examine how RCMV infection affects arterial remodeling and intimal hyperplasia, we measured the crosssectional area and circumference of the intima and media in the tissue sections and counted the number of cell nuclei counterstained with hematoxylin and cells of SM$\alpha$-actin phenotype in both media and intima (Figure 1A). We used the isograft control to confirm previous findings that allogenic response is necessary to initiate graft remodeling $[36,39]$. Neither uninfected nor infected isograft controls collected at 2 and 8 weeks post transplantation developed intimal hyperplasia (Figure 1A). At 8 weeks, the intimal cross-sectional area was threefold thicker in infected allografts $(p<0.05)$ (Figure 1B) and contained 2.5 -fold more cells $(p<0.05)$ compared to uninfected allografts. Also the number of cells was increased in the intima of RCMV-infected allografts at 8 weeks after transplantation compared to uninfected allografts at the same time point. Our findings indicate that RCMV increased intimal hyperplasia in the allografts and contributed to vascular remodeling. 


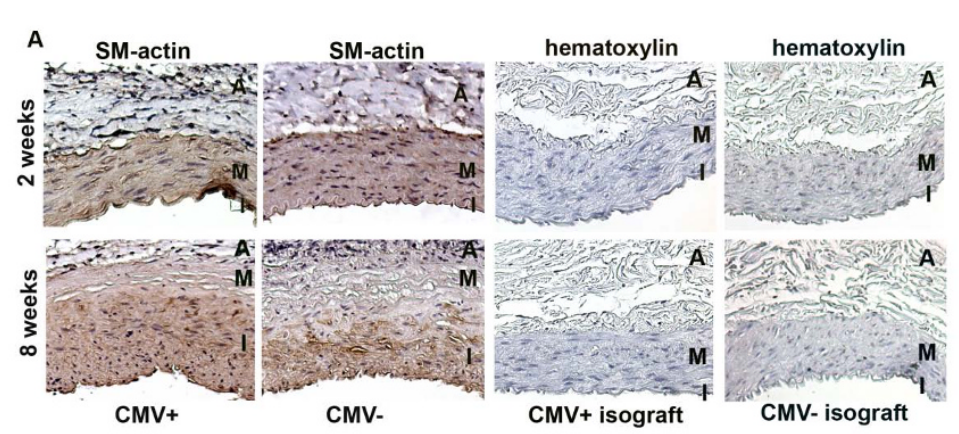

B
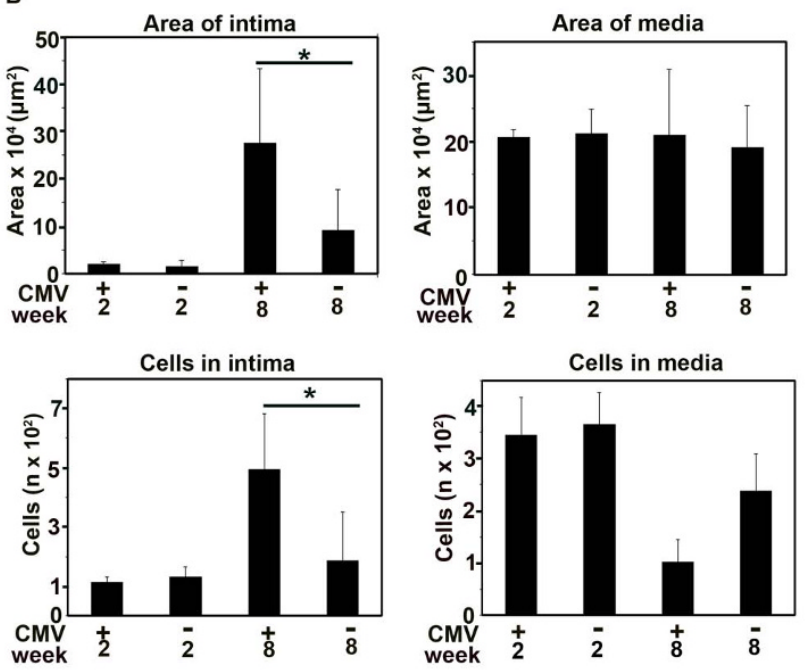

Figure 1 RCMV infection of aortic allografts enhances intimal hyperplasia and contributes to vessel remodelling. (A) The figure presents fragments of cross sections of RCMV-infected and uninfected allografts (controls) collected at 2 and 8 weeks after transplantation and stained for SM- $\alpha$-actin (SM-actin) (Columns 1 and 2). Columns 3 and 4 show fragments of cross sections of the RCMV-infected and uninfected isograft controls at 2 and 8 weeks after transplantation to visualize that there was no intimal formation either in the infected or uninfected isografts. Blue, nuclei counterstained with hematoxylin. Brown, cells positive for SM- $\alpha$-actin. I, intima, M, media, A, adventitia. (B) Bar charts show areas of intima and media presented as cross-sectional area $\left(\mu \mathrm{m}^{2}\right.$ ) (upper panels) and the number of cells per cross section in the intima and media (lower panels) in RCMV-infected and uninfected allografts. At 2 weeks post transplantation the number of cells in the intima and media, and thickness of both layers were similar in infected and uninfected allografts. At 8 weeks, the intima was 3-fold thicker in infected vs. uninfected allografts and contained 2.5fold more cells. The media of infected allografts had fewer cells at 8 week after transplantation. ${ }^{*} p<0.05$ vs. control at 2 and 8 weeks, respectively.

RCMV did not affect the media cross-sectional area, but infected grafts had fewer cells in the media (Figure 1B). To further examine whether this loss of medial cells could be attributed to an enhanced RCMV-induced apoptosis, we double stained RCMV-infected- and uninfected allografts for SM- $\alpha$-actin and caspase 3 and found that apoptosis of SMCs was significantly increased in the media of infected allografts compared to uninfected controls both at 2 weeks $(p<0.001)$, and 8 weeks after transplantation $(p<$ 0.05) (Figure 2).

To further assess the effects of RCMV infection on the extracellular matrix (ECM) components, we examined collagen deposition (Figure 3A). At 2 weeks post transplantation, similar amounts of collagen were present in infected and uninfected allografts. At 8 weeks, RCMV-infected allografts had less collagen $(p<0.05)$ (Figure 3A), indicating that the virus directly or indirectly, affected collagen turnover in the allografts. Therefore, we determined the expression of two matrix metalloproteinases (MMPs) that are major regulators of collagen turnover in the vessel wall, MMP-2 and MMP-9 (Figure 3B and 3C). In infected grafts, MMP-2 expression was confined to the adventitia early after transplantation but was later detected in a diffuse pattern in the whole vessel wall and was significantly increased $(p<0.001)$ compared to uninfected allograft (Figure 3B). MMP-9 expression was not affected by RCMV infection (Figure 3C).

\section{RCMV infection of aortic allografts induces widespread inflammation in the intima and adventitia}

Local RCMV infection resulted in a widespread inflammation of the intima and adventitia early after transplantation 

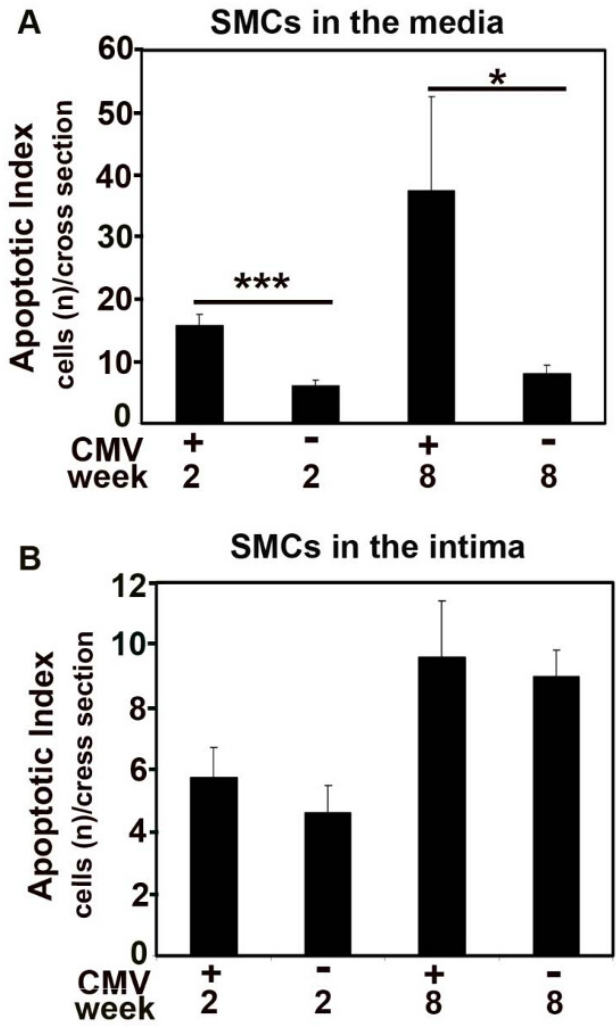

Figure 2 RCMV induces apoptosis of SMCs in the media layer of the aortic allografts. Bar charts show the Apoptotic Index of SMCs presented as the number of apoptotic SMCs per cross section in media (A) and intima (B) of infected and uninfected allografts (controls). Apoptotic SMCs were detected with double

immunostaining for $S M-\alpha$-actin and caspase 3. Both at 2 and 8 weeks after transplantation there was a significant increase of apoptotic SMCs in the media of infected allografts vs. uninfected controls. ${ }^{*} p<0.05$ vs. control at 8 weeks. ${ }^{* *} p<0.001$ vs. control at 2 weeks.

(Figure 4A). To further characterize the phenotypes of infiltrating cells in RCMV-infected rat aortic allografts, we determined the presence of CD68-positive macrophages, CD45-positive leukocytes and CD3-positive lymphocytes (Figure 4B, D, E). Interestingly, most of the infiltrating cells were CD68-positive macrophages (Figure $4 \mathrm{~B}$ ) and $80 \%$ of infiltrating macrophages were RCMV-infected at 8 weeks post transplantation (Figure 4C). CD3-positive lymphocytes were only few and mainly localized in the adventitia and subendothelial area of the allografts at 2 weeks post transplantation (Figure 4E). Moreover, infiltration of CD68-positive macrophages into the intima was fourfold higher in RCMV-infected allografts compared to uninfected controls, both 2 weeks $(p<0.001)$ and 8 weeks $(p<0.01)$ after transplantation (Figure 4F). In the media of infected allografts the number of CD68-positive macrophages was twofold higher at 8 weeks compared to uninfected controls $(p<0.05)$. In the adventitia, there was a massive infiltration of CD68-positive macrophages, with a higher extent in the infected allografts. We observed no significant effect of RCMV on infiltration of CD45-positive leukocytes to the allografts (Figure $4 \mathrm{~F}$ ). These findings indicate that RCMV induced a strong inflammatory response consisting of mainly CD68-positive macrophages in the allografts early and late after transplantation, which likely influenced allograft remodeling.

RCMV induces MCP-1 production in aortic allografts and recruits cells from infected adventitia to the intima

Adventitial progenitor cells appear to be important in vessel remodeling [40-43]. Recently, we found that MCP-1 is a chemoattractant for vascular progenitor cells in cardiac allografts and that their recruitment to the allograft was increased by inflammation [11].

To further examine whether RCMV affected migration of adventitial cells in the rat allograft we first examined if MCP-1, which is a key chemokine responsible for migration of vascular progenitor cells, was altered by RCMV infection. At 2 weeks after transplantation, RCMV infection resulted in a sevenfold increase in the number of MCP-1 positive cells in the allografts $(p<$ 0.001 ) and most of these cells were present in the adventitia (Figure 5A). At 8 weeks, RCMV-infection resulted in a 3.5-fold increase in the number of MCP-1 positive cells, which were present in all vascular layers $(p<0.001)$ (Figure 5A).

To further examine if cells from RCMV-infected adventitia migrated in vivo and thereby could contribute to intimal hyperplasia, we transplanted infected fluorescently labeled isogenic adventitia to the aortic allografts 4 weeks after transplantation, the time point when intimal hyperplasia is emerging [36]. At 2 weeks post adventitia transplantation, we detected fluorescently labeled cells in the intima (Figure 5B). Thus, RCMV induced MCP-1 expression in vivo and induced migration of adventitial cells to the intima.

\section{Discussion}

CMV is thought to be a key pathogen involved in the pathogenesis of TV in human allografts. To evaluate the direct effect of CMV infection on vascular biology, we used a rat model in which aortic allografts were infected ex vivo with RCMV prior to transplantation. We chose 2 time points after transplantation to investigate the development of TV, 2 weeks that represented the early phase of TV in rats; and 8 weeks, when TV was fully developed. We found that RCMV influenced vascular remodeling by increased apoptosis of $\mathrm{SM}-\alpha$-actin 

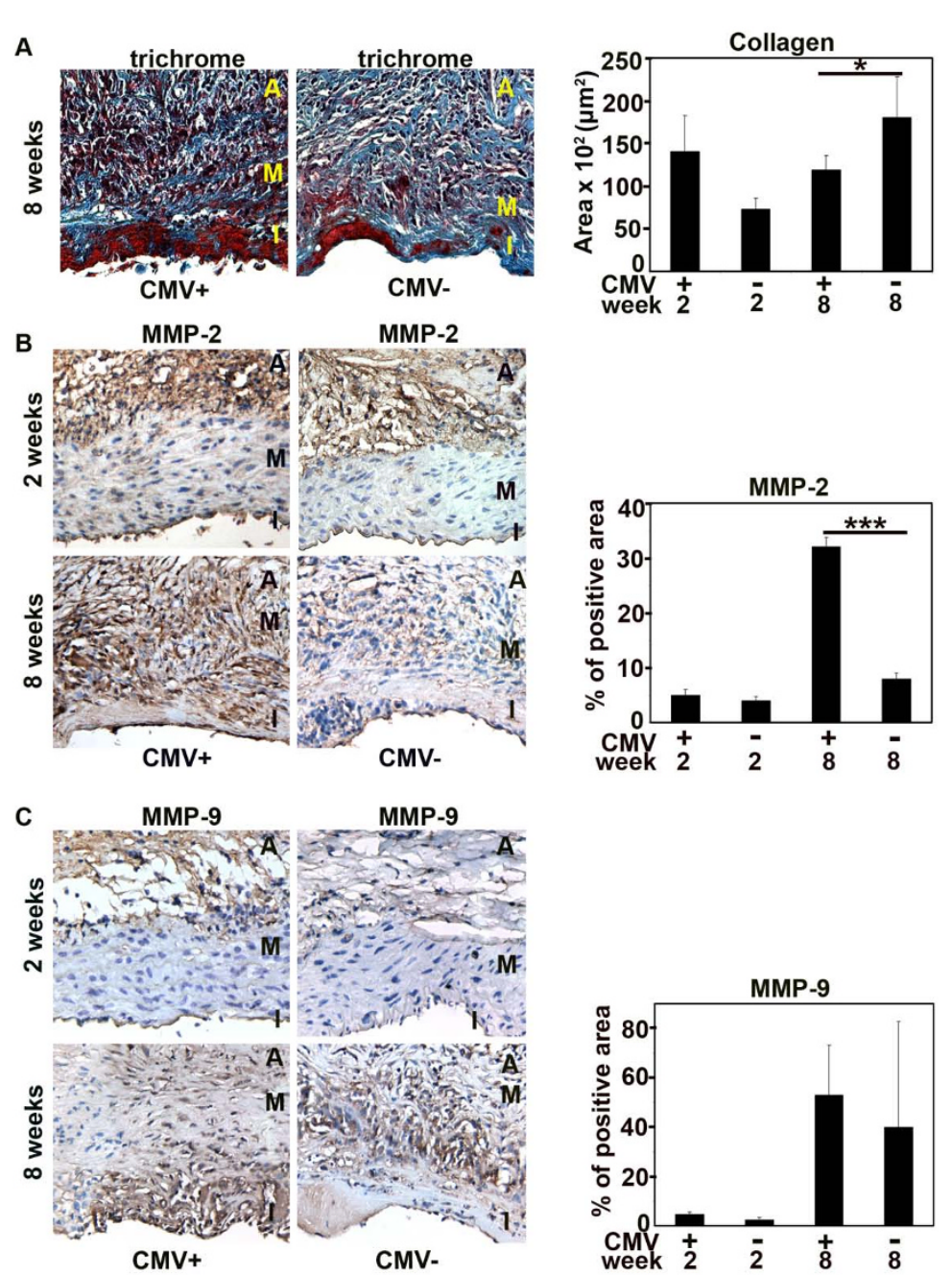

Figure 3 RCMV increases expression of matrix metalloproteinases and decreases collagen deposition in the aortic allografts. (A) Cross sections of RCMV-infected and uninfected allografts (controls) at 2 and 8 weeks post transplantation, stained with Masson's trichrome to identify collagen deposits. Blue, collagen; red, muscle fibers; black, nuclei; I, intima; M, media; A, adventitia. Bar chart shows the collagen deposits in the allografts of RCMV-infected and uninfected allografts at 2 and 8 weeks post transplantation presented as the size $\left(\mu m^{2}\right)$ of positive blue immunostained area per cross section. ${ }^{*} p<0.05$ vs. uninfected controls 8 weeks after transplantation. At 8 weeks, infected allografts had significantly less collagen. (B) Cross sections of RCMV-infected and uninfected allografts (controls) stained to identify MMP-2. Brown indicates positivity for MMP-2. I, intima; M, media; A, adventitia. Bar chart shows percentage of positive areas for MMP-2 in infected and uninfected allografts at 2 and 8 weeks post transplantation determined as the percentage of positive area of the total cross section area. ${ }^{* * *} p<0.001$ vs. uninfected controls at 8 weeks. (C) Cross sections of RCMV-infected and uninfected allografts (controls) stained to identify MMP-9. Brown indicates positivity for MMP-9. I, intima; M, media; A, adventitia. Bar chart shows percentage of positive areas for MMP-9 in infected and uninfected allografts at 2 and 8 weeks post transplantation. Early after transplantation both MMP-2 and MMP-9 after confined mainly to the adventitia.

positive cells in the media layer, decreased extracellular matrix deposits and increased intimal hyperplasia. Moreover, RCMV induced a strong infiltration of CD68-positive macrophages mainly in the adventitia and resulted in an increase of MCP-1 in the allograft, which resulted in migration of adventitial cells towards the intima that most likely also contributed to intimal hyperplasia.

Vessel stability is sustained by a balance between cellular proliferation and apoptosis, and the synthesis or degradation of extracellular matrix (ECM) components.
Alterations in this balance have been shown to contribute to the development of vascular diseases. Previous studies suggested that HCMV infection negatively influenced coronary artery remodeling in the first year after heart transplantation [12]. Moreover, systemic RCMV infection stimulated arterial SMCs proliferation when vascular injury was induced in the rat model of restenosis [44], although in this particular study the ECM content in the injured artery was not determined. 


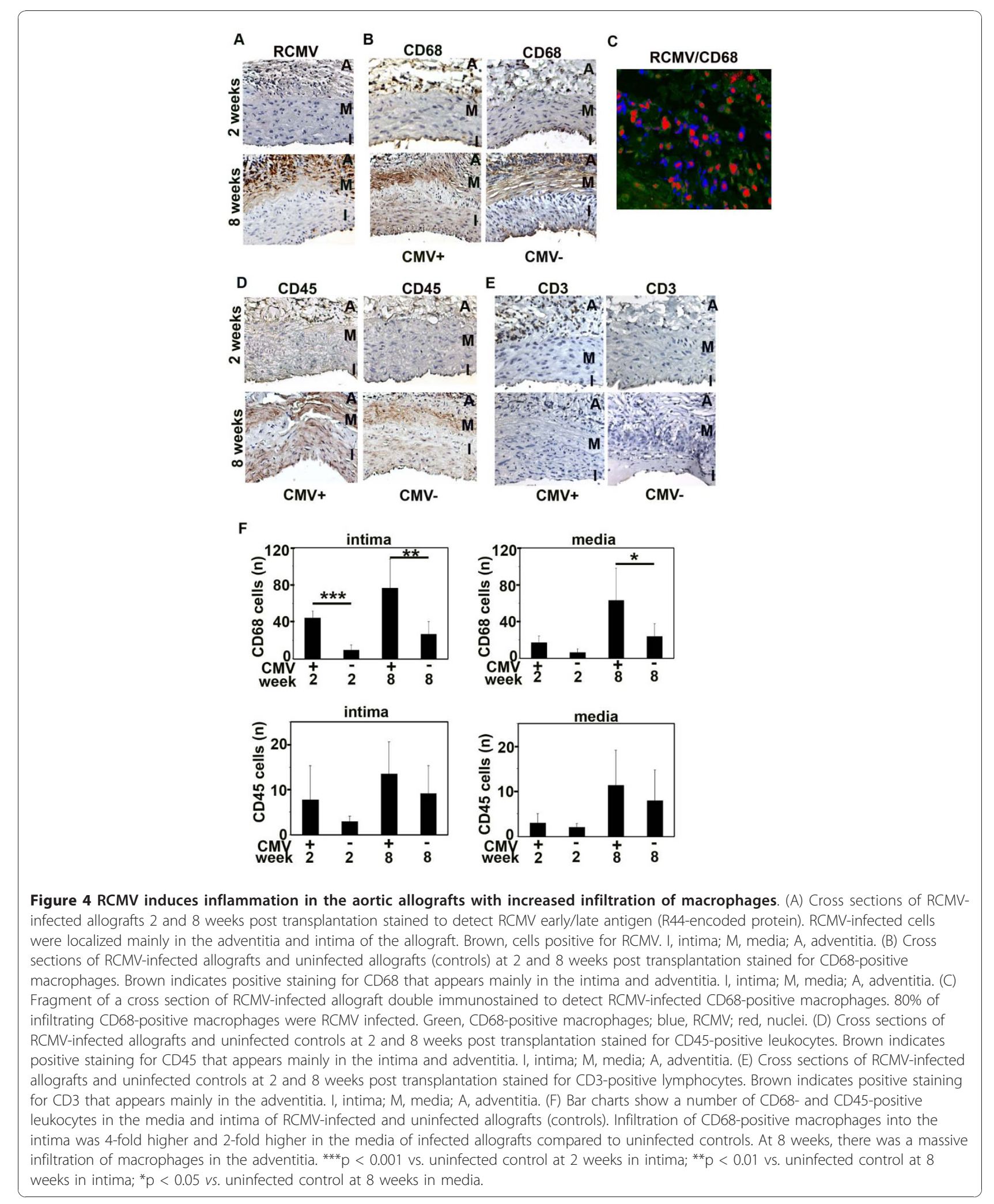




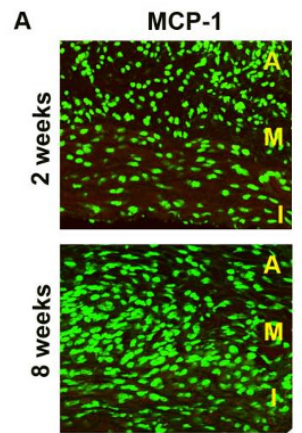

$\mathrm{CMV+}$
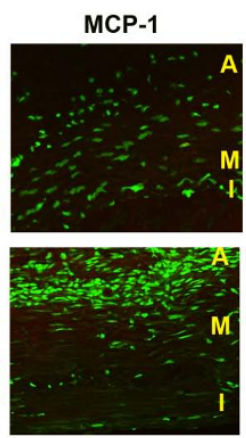

CMV-

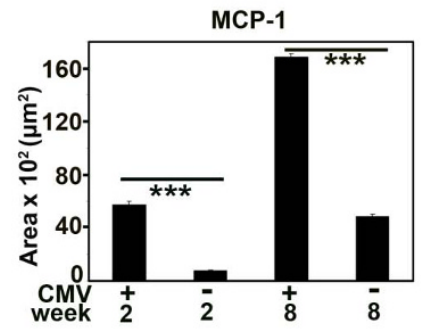

RCMV-infected allograft
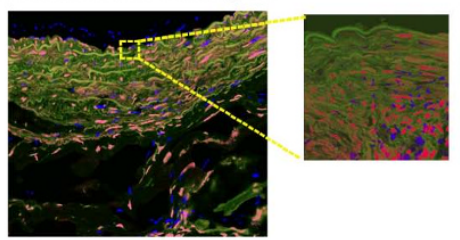

Figure 5 RCMV increases expression of MCP-1 in the aortic allografts and cells from the infected adventitia migrate towards the intima. (A) Cross sections of the RCMV-infected and uninfected allografts (controls) at 2 and 8 weeks post transplantation stained for MCP-1. Green, MCP-1. Bar chart shows MCP-1 expression in the allografts, presented as positive area of the cross section $\left(\mu m^{2}\right)$. Both at 2 and 8 weeks after transplantation MCP-1 expression was increased in infected allografts (7-fold at 2 weeks and 3.5-fold at 8 weeks post transplantation).

${ }^{* * *} p<0.001$ vs. uninfected control at 2 and 8 weeks, respectively. I, intima; $M$, media; A, adventitia. (B) Cross section of the aortic allograft with transplanted fluorescently labeled isogenic adventitial graft. Grafts were collected 2 weeks post adventitia transplantation. Picture shows labeled blue adventitial cells in the allograft intima. Green, SM-actin; Blue, migrated adventitial cells; Red, nuclei.

In our study, we found that RCMV increased apoptosis of SM- $\alpha$-actin positive cells in the media, which contributed to an earlier and enhanced destruction of the medial layer after transplantation compared to uninfected allografts. This destruction of the media layer led to further fibrosis and stimulation of intimal hyperplasia. RCMV increased proliferation of SM- $\alpha$-actin positive cells in the intima resulting in intimal thickening in TV. Consistent with other studies, we observed no intimal formation in isogenic grafts (isografts) $[22,36,45]$, even if they were infected with CMV $[27,31,35]$, which suggests that allogenic response is necessary for CMV to further trigger cellular responses in TV.

Collagen is the major component of the ECM in the vessel wall and is crucial for vessel wall integrity. Here, we found that RCMV decreased the collagen content in the allografts late after transplantation. Moreover, expression of MMP-2, which regulates collagen turnover in the vessel wall and is thought to be altered in vascular diseases $[46,47]$ was upregulated in infected allografts. These findings are in agreement with earlier report showing that the expression of proteins involved in MMP activation and ECM modification are induced in RCMV-infected cardiac allografts as TV develops [5]. These findings indicate that RCMV directly or by interacting with host immunity reduces the integrity of the artery wall and thereby increases tissue vulnerability to injury, inflammation and uncontrolled remodeling.

Previous studies showed that RCMV induces an early inflammatory response in the adventitia (perivasculitis) and the subendothelial space [26] in a rat aortic transplant model with systemic RCMV infection. However, the types of infected cells in the graft were not identified. In murine models of cardiac transplantation, mouse CMV infection resulted in chronic vascular rejection, intimal thickening, and vascular occlusion [48]. MCP-1 and its receptor (chemokine receptor 2) are though to drive the recruitment of inflammatory cells and cells with $S M-\alpha$-actin phenotype to the sites of vascular injury in atherosclerosis and TV [49]. MCP-1 transcripts have been identified in RCMV-infected rat cardiac allografts affected with TV [5]. Recently we found that MCP-1 is a chemoattractant for progenitor cells migrating to cardiac allografts, which was enhanced by inflammation [11]. We also showed that antibodies to MCP-1 significantly reduced inflammation and accumulation of these cells in the graft.

In our study, we observed RCMV-dependent recruitment of CD68-positive macrophages. The infiltration of lymphocytes into the allograft was mainly confined to 
the adventitia and the subendothelial area early after transplantation. This pattern was also previously observed by us and other authors in the similar animal models of TV $[36,39]$, as well as in the models of RCMV-infected rat aortic and cardiac allografts $[26,50,51]$. The lymphocytic infiltration was observed until 1 week post transplantation [26] but not thereafter and late inflammatory response consisted mainly of macrophages.

We also observed that MCP-1 is highly expressed in infected allografts in areas of the artery with high numbers of macrophages. This scenario may contribute to the pro-inflammatory state in the allograft, resulting in release of inflammatory cytokines and chemokines that accelerate the inflammatory process and sustain further viral replication [21] and vascular remodeling. Importantly, CMV also carries chemokines and chemokine receptor homologues that recruit and stimulate cellular infiltration, and thereby further potentiate the inflammatory response [52]. Furthermore, several studies have shown that infection with viruses can result in the upregulation of the eicosanoid pathways $[53,54]$. A relationship between CMV and enhanced cyclooxygenase-2 (COX-2) expression has been identified both in vitro and in vivo [55,56]. COX-2 and prostaglandin pathways are up-regulated in CMV infected cells and this may be essential for efficient CMV replication [55]. Interestingly, in the CMV-infected SMCs, induced 5-Lipoxygenase expression and increased leukotriene B4 production may promote inflammation through induced infiltration and activation of leukocytes [17,57]. Thus, CMV infection of tissues may exacerbate inflammation and contribute to the observed pathology.

The vascular adventitia has been implied as a source of cells that contribute to intimal hyperplasia $[41,43,58]$ and migrate in the vessel wall in order to localize in vascular lesions [59]. Here, we investigated whether cells derived from infected adventitia migrated to the intima and if migration of adventitial cells was altered by RCMV. We transplanted fluorescently labeled RCMVinfected adventitia to the allografts 4 weeks after the allograft transplantation and found that adventitial cells migrated to the intima and most likely contributed to intimal hyperplasia. Interestingly, we showed that RCMV infection significantly enhanced MCP-1 production in the allograft and thereby created the environment to support cellular migration. Our observation is consistent with our previous finding that expression of the virally encoded chemokine receptor chomologue, US28 in the presence of CC chemokines, including MCP-1, was sufficient to promote cellular migration [21]. Moreover, it suggests that the virus itself creates pro-inflammatory environment and is able to alter cellular processes involved in the development of TV.

\section{Conclusions}

In summary, acute RCMV infection of allografts led to enhanced MCP-1 production and massive infiltration of macrophages, following transplantation, resulting in inflammation and vascular remodeling that contributed to more rapid and severe vessel narrowing. Our findings increase understanding of the CMV role in vessel remodeling and highlight the importance of managing CMV infections in transplant patients.

\section{Acknowledgements}

We thank Prof. Cathrien A. Bruggeman from Maastricht University, The Netherlands for providing us with the RCMV Maastricht strain. This work was supported by The Swedish Heart and Lung Foundation (20070446), The Swedish Medical Research Council 2009-10274-68890-53; K 2010-56X-1261513-3), The Linneus Center for Research on Inflammation and Cardiovascular Disease of the Swedish Research Council, The Polish Science Research Committee (MN3B138), Stockholm County Council (20080240).

\section{Author details}

${ }^{1}$ Experimental Cardiovascular Research Unit, Department of Medicine, Solna, Karolinska Institutet, Stockholm, Sweden. ${ }^{2}$ Department of General, Vascular and Oncologic Surgery, Medical University of Warsaw, Warsaw, Poland. ${ }^{3}$ Department of General Biochemistry and Nutrition, Medical University of Warsaw, Warsaw, Poland. ${ }^{4}$ Maastricht University Medical Center, Maastricht, The Netherlands.

\section{Authors' contributions}

MKG participated in the design of the study, performed the experiments, analyzed the data and wrote the manuscript. BK participated in the design of the study and performed the experiments. JS participated in the design of the study and performed the experiments. FS analyzed the data and helped to draft the manuscript. CSN participated in the study design, analyzed the data and assisted in writing the manuscript. PR participated in the study design and helped to analyze the data. All authors read and approved the final manuscript.

\section{Competing interests}

The authors declare that they have no competing interests.

Received: 9 September 2010 Accepted: 23 December 2010 Published: 23 December 2010

\section{References}

1. Melnick JL, Petrie BL, Dreesman GR, Burek J, McCollum CH, DeBakey ME: Cytomegalovirus antigen within human arterial smooth muscle cells. Lancet 1983, 2(8351):644-7.

2. Speir $E$, Modali R, Huang ES, et al: Potential role of human cytomegalovirus and p53 interaction in coronary restenosis. Science 1994, 265(5170):391-4.

3. Vainas T, Stassen FR, Bruggeman CA, et al: Synergistic effect of Toll-like receptor 4 and $C D 14$ polymorphisms on the total atherosclerosis burden in patients with peripheral arterial disease. J Vasc Surg 2006, 44:326-32.

4. Bentz GL, Yurochko AD: Human CMV infection of endothelial cells induces an angiogenic response through viral binding to EGF receptor and beta1 and beta3 integrins. Proc Natl Acad Sci USA 2008, 105(14):5531-6.

5. Streblow DN, Kreklywich CN, Andoh T, et al: The role of angiogenic and wound repair factors during CMV-accelerated transplant vascular sclerosis in rat cardiac transplants. Am J Transplant 2008, 8(2):277-87.

6. Burnett MS, Durrani $S$, Stabile $E$, et al: Murine cytomegalovirus infection increases aortic expression of proatherosclerotic genes. Circulation 2004, 109(7):893-7.

7. Cramer DV, Wu GD, Chapman FA, Cajulis E, Wang HK, Makowka L: Lymphocytic subsets and histopathologic changes associated with the development of heart transplant arteriosclerosis. J Heart Lung Transplant 1992, 11(3 Pt 1):458-66. 
8. Edlin RS, Tsai S, Yamanouchi D, Wang C, Liu B, Kent KC: Characterization of primary and restenotic atherosclerotic plaque from the superficial femoral artery: Potential role of Smad3 in regulation of SMC proliferation. J Vasc Surg 2009, 49(5):1289-95.

9. Csanyi G, Taylor WR, Pagano PJ: NOX and inflammation in the vascular adventitia. Free Radic Biol Med 2009, 47(9):1254-66.

10. Maiellaro K, Taylor WR: The role of the adventitia in vascular inflammation. Cardiovasc Res 2007, 75(4):640-8.

11. Religa P, Grudzinska MK, Bojakowski K, et al: Host-derived smooth muscle cells accumulate in cardiac allografts: role of inflammation and monocyte chemoattractant protein 1. PLOS One 2009, 4(1):e4187.

12. Potena L, Grigioni F, Ortolani P, et al: Relevance of cytomegalovirus infection and coronary-artery remodeling in the first year after heart transplantation: a prospective three-dimensional intravascular ultrasound study. Transplantation 2003, 75(6):839-43.

13. Soderberg-Naucler $C$, Nelson JY: Human cytomegalovirus latency and reactivation - a delicate balance between the virus and its host's immune system. Intervirology 1999, 42(5-6):314-21.

14. Hansson GK, Robertson AK, Soderberg-Naucler C: Inflammation and atherosclerosis. Annu Rev Pathol 2006, 1:297-329.

15. Soderberg-Naucler C: HCMV microinfections in inflammatory diseases and cancer. J Clin Virol 2008, 41(3):218-23.

16. Vliegen I, Herngreen SB, Grauls GE, Bruggeman CA, Stassen FR: Mouse cytomegalovirus antigenic immune stimulation is sufficient to aggravate atherosclerosis in hypercholesterolemic mice. Atherosclerosis 2005 , 181(1):39-44.

17. Qiu H, Straat K, Rahbar A, Wan M, Soderberg-Naucler C, Haeggstrom JZ: Human CMV infection induces 5-lipoxygenase expression and leukotriene B4 production in vascular smooth muscle cells. J Exp Med 2008, 205(1):19-24.

18. Hendrix MG, Daemen M, Bruggeman CA: Cytomegalovirus nucleic acid distribution within the human vascular tree. Am J Pathol 1991, 138(3):563-7.

19. Hendrix MG, Salimans MM, van Boven CP, Bruggeman CA: High prevalence of latently present cytomegalovirus in arterial walls of patients suffering from grade III atherosclerosis. Am J Pathol 1990, 136(1):23-8.

20. Forster MR, Bickerstaff AA, Wang JJ, Zimmerman PD, Cook CH: Allogeneic stimulation causes transcriptional reactivation of latent murine cytomegalovirus. Transplant Proc 2009, 41(5):1927-31.

21. Streblow DN, Soderberg-Naucler C, Vieira J, et al: The human cytomegalovirus chemokine receptor US28 mediates vascular smooth muscle cell migration. Cell 1999, 99(5):511-20.

22. Streblow DN, Kreklywich CN, Smith P, et al: Rat cytomegalovirusaccelerated transplant vascular sclerosis is reduced with mutation of the chemokine-receptor R33. Am J Transplant 2005, 5(3):436-42.

23. Hillebrands JL, van Dam JG, Onuta G, et al: Cytomegalovirus-enhanced development of transplant arteriosclerosis in the rat; effect of timing of infection and recipient responsiveness. Transpl Int 2005, 18(6):735-42.

24. Kloppenburg G, de Graaf R, Grauls G, Bruggeman CA, van Hooff JP, Stassen F: FK778 attenuates cytomegalovirus-enhanced vein graft intimal hyperplasia in a rat model. Intervirology 2009, 52(4):189-95.

25. Lemstrom K, Koskinen P, Krogerus L, Daemen M, Bruggeman C, Hayry P: Cytomegalovirus antigen expression, endothelial cell proliferation, and intimal thickening in rat cardiac allografts after cytomegalovirus infection. Circulation 1995, 92(9):2594-604.

26. Lemstrom KB, Aho PT, Bruggeman CA, Hayry PJ: Cytomegalovirus infection enhances mRNA expression of platelet-derived growth factor-BB and transforming growth factor-beta 1 in rat aortic allografts. Possible mechanism for cytomegalovirus-enhanced graft arteriosclerosis Arterioscler Thromb 1994, 14(12):2043-52.

27. Lemstrom KB, Bruning JH, Bruggeman CA, Lautenschlager IT, Hayry PJ: Cytomegalovirus infection enhances smooth muscle cell proliferation and intimal thickening of rat aortic allografts. J Clin Invest 1993, 92(2):549-58.

28. Streblow DN, van Cleef KW, Kreklywich CN, et al: Rat cytomegalovirus gene expression in cardiac allograft recipients is tissue specific and does not parallel the profiles detected in vitro. J Virol 2007, 81(8):3816-26.

29. Lautenschlager I, Soots A, Krogerus L, et al: Effect of cytomegalovirus on an experimental model of chronic renal allograft rejection under tripledrug treatment in the rat. Transplantation 1997, 64(3):391-8.
30. Cook CH, Bickerstaff AA, Wang JJ, et al: Disruption of murine cardiac allograft acceptance by latent cytomegalovirus. Am J Transplant 2009, 9(1):42-53.

31. Heim C, Abele-Ohl S, Eckl S, et al: Murine cytomegalovirus infection leads to increased levels of transplant arteriosclerosis in a murine aortic allograft model. Transplantation 2010, 90(4):373-9.

32. Li FL, Grauls G, Yin M, Bruggeman CA: Correlation between the intensity of cytomegalovirus infection and the amount of perivasculitis in aortic allografts. Transpl Int 1996, 9(Suppl 1):S340-4.

33. Bruggeman CA, Meijer $H$, Bosman F, van Boven CP: Biology of rat cytomegalovirus infection. Intervirology 1985, 24(1):1-9.

34. Onuta G, van Ark J, Rienstra H, et al: Development of transplant vasculopathy in aortic allografts correlates with neointimal smooth muscle cell proliferative capacity and fibrocyte frequency. Atherosclerosis 2010, 209:393-402

35. Orloff SL, Yin Q, Corless CL, Orloff MS, Rabkin JM, Wagner CR: Tolerance induced by bone marrow chimerism prevents transplant vascular sclerosis in a rat model of small bowel transplant chronic rejection. Transplantation 2000, 69(7):1295-303.

36. Bojakowski K, Religa P, Bojakowska M, Hedin U, Gaciong Z, Thyberg J: Arteriosclerosis in rat aortic allografts: early changes in endothelial integrity and smooth muscle phenotype. Transplantation 2000, 70(1):65-72.

37. Religa P, Bojakowski K, Bojakowska M, Gaciong Z, Thyberg J, Hedin U: Allogenic immune response promotes the accumulation of host-derived smooth muscle cells in transplant arteriosclerosis. Cardiovasc Res 2005, 65(2):535-45.

38. Campbell MT, Hile KL, Zhang H, et al: Toll-Like Receptor 4: A Novel Signaling Pathway During Renal Fibrogenesis. J Surg Res 2009

39. Mennander A, Tiisala S, Halttunen J, Yilmaz S, Paavonen T, Hayry P: Chronic rejection in rat aortic allografts. An experimental model for transplant arteriosclerosis. Arterioscler Thromb 1991, 11(3):671-80.

40. Siow RC, Churchman AT: Adventitial growth factor signalling and vascular remodelling: potential of perivascular gene transfer from the outside-in. Cardiovasc Res 2007, 75(4):659-68.

41. Hu Y, Zhang Z, Torsney E, et al: Abundant progenitor cells in the adventitia contribute to atherosclerosis of vein grafts in ApoE-deficient mice. J Clin Invest 2004, 113(9):1258-65.

42. Matsumoto Y, Hof A, Baumlin Y, Muller M, Prescott MF, Hof RP: Dynamics of medial smooth muscle changes after carotid artery transplantation in transgenic mice expressing green fluorescent protein. Transplantation 2003, 76(11):1569-72.

43. Shi Y, O'Brien JE, Fard A, Mannion JD, Wang D, Zalewski A: Adventitial myofibroblasts contribute to neointimal formation in injured porcine coronary arteries. Circulation 1996, 94(7):1655-64.

44. Kloppenburg G, de Graaf R, Herngreen S, Grauls G, Bruggeman C, Stassen F: Cytomegalovirus aggravates intimal hyperplasia in rats by stimulating smooth muscle cell proliferation. Microbes Infect 2005, 7(2):164-70.

45. Religa P, Bojakowski K, Gaciong Z, Thyberg J, Hedin U: Arteriosclerosis in rat aortic allografts: dynamics of cell growth, apoptosis and expression of extracellular matrix proteins. Mol Cell Biochem 2003, 249(1-2):75-83.

46. Lim CS, Shalhoub J, Gohel MS, Shepherd AC, Davies AH: Matrix metalloproteinases in vascular disease-a potential therapeutic target? Curr Vasc Pharmacol 2010, 8(1):75-85.

47. Hakuno D, Kimura N, Yoshioka M, et al: Periostin advances atherosclerotic and rheumatic cardiac valve degeneration by inducing angiogenesis and MMP production in humans and rodents. J Clin Invest 2010, 120(7):2292-306

48. Bolinger B, Engeler D, Krebs P, et al: IFN-gamma-receptor signaling ameliorates transplant vasculopathy through attenuation of CD8+ T-cell-mediated injury of vascular endothelial cells. Eur J Immunol 40(3):733-43.

49. Ni W, Kitamoto S, Ishibashi M, et al: Monocyte chemoattractant protein-1 is an essential inflammatory mediator in angiotensin II-induced progression of established atherosclerosis in hypercholesterolemic mice. Arterioscler Thromb Vasc Biol 2004, 24(3):534-9.

50. Koskinen P, Lemstrom K, Bruggeman C, Lautenschlager I, Hayry P: Acute cytomegalovirus infection induces a subendothelial inflammation (endothelialitis) in the allograft vascular wall. A possible linkage with enhanced allograft arteriosclerosis. Am J Pathol 1994, 144(1):41-50. 
51. Lemstrom KB, Bruning JH, Bruggeman CA, et al: Cytomegalovirus infection-enhanced allograft arteriosclerosis is prevented by DHPG prophylaxis in the rat. Circulation 1994, 90(4):1969-78.

52. Noda S, Aguirre SA, Bitmansour A, et al: Cytomegalovirus MCK-2 controls mobilization and recruitment of myeloid progenitor cells to facilitate dissemination. Blood 2006, 107(1):30-8.

53. Rue CA, Jarvis MA, Knoche AJ, et al: A cyclooxygenase-2 homologue encoded by rhesus cytomegalovirus is a determinant for endothelial cell tropism. J Virol 2004, 78(22):12529-36.

54. Steer SA, Corbett JA: The role and regulation of COX-2 during viral infection. Viral Immunol 2003, 16(4):447-60.

55. Zhu H, Cong JP, Yu D, Bresnahan WA, Shenk TE: Inhibition of cyclooxygenase 2 blocks human cytomegalovirus replication. Proc Natl Acad Sci USA 2002, 99(6):3932-7.

56. Browne EP, Wing B, Coleman D, Shenk T: Altered cellular mRNA levels in human cytomegalovirus-infected fibroblasts: viral block to the accumulation of antiviral mRNAs. J Virol 2001, 75(24):12319-30.

57. Luster $A D$, Tager $A M: T$-cell trafficking in asthma: lipid mediators grease the way. Nat Rev Immunol 2004, 4(9):711-24.

58. Ji J, Xu F, Li L, Chen R, Wang J, Hu WC: Activation of adventitial fibroblasts in the early stage of the aortic transplant vasculopathy in rat. Transplantation 89(8):945-53.

59. Mayr M, Zampetaki A, Sidibe A, et al: Proteomic and metabolomic analysis of smooth muscle cells derived from the arterial media and adventitial progenitors of apolipoprotein E-deficient mice. Circ Res 2008, 102(9):1046-56.

doi:10.1186/2042-4280-1-7

Cite this article as: Grudzinska et al:: RCMV increases intimal hyperplasia by inducing inflammation, MCP-1 expression and recruitment of adventitial cells to intima. Herpesviridae 2010 1:7.

\section{Submit your next manuscript to BioMed Central and take full advantage of:}

- Convenient online submission

- Thorough peer review

- No space constraints or color figure charges

- Immediate publication on acceptance

- Inclusion in PubMed, CAS, Scopus and Google Scholar

- Research which is freely available for redistribution

Submit your manuscript at www.biomedcentral.com/submit
Biomed Central 